\title{
Specific contributions of basal ganglia and cerebellum to the neural tracking of rhythm
}

Citation for published version (APA):

Nozaradan, S., Schwartze, M., Obermeier, C., \& Kotz, S. A. (2017). Specific contributions of basal ganglia and cerebellum to the neural tracking of rhythm. Cortex, 95, 156-168.

https://doi.org/10.1016/j.cortex.2017.08.015

Document status and date:

Published: 01/10/2017

DOI:

10.1016/j.cortex.2017.08.015

Document Version:

Publisher's PDF, also known as Version of record

Document license:

Taverne

Please check the document version of this publication:

- A submitted manuscript is the version of the article upon submission and before peer-review. There can be important differences between the submitted version and the official published version of record.

People interested in the research are advised to contact the author for the final version of the publication, or visit the DOI to the publisher's website.

- The final author version and the galley proof are versions of the publication after peer review.

- The final published version features the final layout of the paper including the volume, issue and page numbers.

Link to publication

\footnotetext{
General rights rights.

- You may freely distribute the URL identifying the publication in the public portal. please follow below link for the End User Agreement:

www.umlib.nl/taverne-license

Take down policy

If you believe that this document breaches copyright please contact us at:

repository@maastrichtuniversity.nl

providing details and we will investigate your claim.
}

Copyright and moral rights for the publications made accessible in the public portal are retained by the authors and/or other copyright owners and it is a condition of accessing publications that users recognise and abide by the legal requirements associated with these

- Users may download and print one copy of any publication from the public portal for the purpose of private study or research.

- You may not further distribute the material or use it for any profit-making activity or commercial gain

If the publication is distributed under the terms of Article $25 \mathrm{fa}$ of the Dutch Copyright Act, indicated by the "Taverne" license above, 


\title{
Research Report
}

\section{Specific contributions of basal ganglia and cerebellum to the neural tracking of rhythm}

\author{
Sylvie Nozaradan ${ }^{a, b, c, *}$, Michael Schwartze ${ }^{d, e}$, Christian Obermeier ${ }^{d}$ and \\ Sonja A. Kotz ${ }^{\text {d,e }}$ \\ a MARCS Institute for Brain, Behavior and Development, Western Sydney University, Australia \\ ${ }^{\mathrm{b}}$ Institute of Neuroscience (Ions), Université Catholique de Louvain (UCL), Belgium \\ ${ }^{\mathrm{c}}$ International Laboratory for Brain, Music and Sound Research (BRAMS), Montreal, Canada \\ ${ }^{\mathrm{d}}$ Max Planck Institute for the Human Cognitive and Brain Sciences, Dept. of Neuropsychology, Leipzig, Germany \\ e Basic and Applied NeuroDynamics Laboratory, Faculty of Psychology and Neuroscience, Dept. of Neuropsychology \\ and Psychopharmacology, Maastricht University, Maastricht, The Netherlands
}

\section{A R T I C L E I N F O}

Article history:

Received 25 February 2017

Reviewed 4 July 2017

Revised 16 July 2017

Accepted 7 August 2017

Action editor Lesley Fellows

Published online 19 August 2017

\section{Keywords:}

Musical rhythm

Frequency-tagging

EEG

Human brain lesion

Basal ganglia

Cerebellum

Auditory processing

\begin{abstract}
A B S T R A C T
How specific brain networks track rhythmic sensory input over time remains a challenge in neuroimaging work. Here we show that subcortical areas, namely the basal ganglia and the cerebellum, specifically contribute to the neural tracking of rhythm. We tested patients with focal lesions in either of these areas and healthy controls by means of electroencephalography (EEG) while they listened to rhythmic sequences known to induce selective neural tracking at a frequency corresponding to the most-often perceived pulse-like beat. Both patients and controls displayed neural responses to the rhythmic sequences. However, these response patterns were different across groups, with patients showing reduced tracking at beat frequency, especially for the more challenging rhythms. In the cerebellar patients, this effect was specific to the rhythm played at a fast tempo, which places high demands on the temporally precise encoding of events. In contrast, basal ganglia patients showed more heterogeneous responses at beat frequency specifically for the most complex rhythm, which requires more internal generation of the beat. These findings provide electrophysiological evidence that these subcortical structures selectively shape the neural representation of rhythm. Moreover, they suggest that the processing of rhythmic auditory input relies on an extended corticosubcortico-cortical functional network providing specific timing and entrainment sensitivities.
\end{abstract}

(C) 2017 Elsevier Ltd. All rights reserved.

\section{Introduction}

Auditory perception excels in tracking rhythmic features of the sensory input over time (Joris, Schreiner, \& Rees, 2004).
However, the actual neural substrate of this rhythmic process remains largely unknown. Although auditory cortical activity faithfully tracks rhythmic temporal fluctuations of acoustic input (e.g. Brugge et al., 2009; Ding, Melloni, Zhang, Tian, \&

\footnotetext{
* Corresponding author. MARCS Institute for Brain, Behaviour and Development, Western Sydney University, Locked Bag 1797, Penrith, NSW 2751, Australia.

E-mail address: sylvie.nozaradan@uclouvain.be (S. Nozaradan). 
Poeppel, 2016; Eggermont, 2001; Gourévitch, Le Bouquin Jeannès, Faucon, \& Liégeois-Chauvel, 2008; Liégeois-Chauvel, Lorenzi, Trébuchon, Régis, \& Chauvel, 2004; Pantev et al., 1988; Picton, Skinner, Champagne, Kellett, \& Maiste, 1987), this neural activity may constitute the final product of preceding and interactive processing stages (Large, Herrera, \& Velasco, 2015; Patel \& Iversen, 2014). More specifically, auditory cortices may interact with subcortical areas that have been shown to engage in temporal processing such as the basal ganglia and the cerebellum. The basal ganglia are claimed to play a key role in predicting upcoming events on the basis of a relative timing mechanism, possibly supporting the internal generation of a periodic pulse-like beat when listening to musical rhythms (Grahn and Brett, 2009; Grahn, 2009; Teki, Grube, Kumar, \& Griffiths, 2011; Schwartze, Keller, Patel, \& Kotz, 2011; Schwartze \& Kotz, 2013). In contrast, the cerebellum is considered to play a critical role in absolute duration based mechanisms (Grube, Cooper, Chinnery, \& Griffiths, 2010; Grube, Lee, Griffiths, Barker, Woodruff, \& 2010; Knolle, Schröger, Baess, \& Kotz, 2012; Knolle, Schröger, \& Kotz, 2013; Teki et al., 2011; Teki, Grube, \& Griffiths, 2012) and in the encoding of the precise timing of events particularly in the subsecond range (Ivry, Keele, \& Diener, 1988; Ivry \& Keele, 1989; Ivry \& Schlerf, 2008).

The main goal of the current study was to specify the role of the basal ganglia and the cerebellum in the neural tracking of rhythmic streams. Uncovering these mechanisms would provide important insight into the operating principles of a cortico-subcortico-cortical network that has been proposed as the functional substrate of rhythm and timing processes (Schwartze \& Kotz, 2013). To this end we recorded electroencephalographic activity (EEG) from patients with lesions in the basal ganglia or the cerebellum and from healthy matched controls while they listened to different rhythmic sequences. These rhythms were specifically selected on the basis of previous work indicating that particular frequencies of the continuous EEG activity elicited by these rhythms are amplified when they correspond to the frequency of a most-often perceived periodic pulse-like beat, even when a sound does not occur on each beat, i.e. in syncopated rhythms (Nozaradan, Peretz, \& Mouraux, 2012b; Nozaradan, 2014; Nozaradan et al., 2016a). These rhythms were presented at different rates, either well within the musical tempo range promoting beat perception $(<5 \mathrm{~Hz})$ or at the upper limit of this range as such faster tempi requires the perceptual grouping of events into larger number of events to entrain to the beat (Nozaradan et al., 2012b; Repp, 2005).

First, neural tracking of the rhythm was compared across patients and controls based on a frequency-tagging approach measuring the amplitude of the responses expected at frequencies corresponding to the frequency components of the rhythmic contour of the sequences (Nozaradan, 2014). The average amplitude of these frequency-tagged responses provides an index for the general capacity of the auditory system to respond to the sequences, regardless of the relative amplitude of each frequency component elicited by the rhythmic sequences. Most importantly, while we did not expect to find differences in this average amplitude of the responses across the different groups of participants, we hypothesized to find finer-grained differences in these response patterns, in the form of an altered selective neural tracking at beat frequency in patients compared to controls. Specifically, we predicted basal ganglia patients to show reduced relative amplitude at beat frequency, especially for a more complex rhythm, in which a sound does not systematically coincide with a beat, thus relying more on endogenous beat generation. In contrast, we predicted cerebellar patients to display reduced relative amplitude at beat frequency, especially for the sequences played at the fastest tempo, as this tempo requires rapid and precise encoding of temporal events and a switch to a slower frequency of perceptual grouping in order to entrain to the beat. This dissociation between the three groups of participants would indicate a specific contribution of each of these subcortical structures to the neural tracking of rhythmic sensory input.

\section{Materials and methods}

\subsection{Participants}

Eleven patients with lesions in the basal ganglia (mean age 50.9 , range $=30-64$ years), eleven patients with lesions in the cerebellum (mean age 52.6, range $=37-64$ years), and eleven healthy controls (mean age 52.1, range $=28-63$ years) took part in the experiment after providing written informed consent. The controls were recruited via a database at the MaxPlanck Institute for Human Cognitive and Brain Sciences (Leipzig, Germany) and matched the patients in age, education (in years), gender (5 males per group), and handedness (all right-handed). Structural neuroimaging techniques (MRI and brain CT) were used to locate and manually delineate lesions in MRIcron, thus generating volumes of interest for each patient (Rorden, Karnath, \& Bonilha, 2007). Focal vascular lesions typically resulted from an ischemic or hemorrhagic stroke that had occurred 9.5 years before on average (see Table 1 for details on the age of the patients, the date of the incident, and on the topography and volume of the lesions). The structural overlay of the lesions is depicted in Fig. 1. Additional lesions outside the cerebellum and basal ganglia were present in some patients (see Table 1). All participants received a compensatory fee. None of the participants were professional musicians or had prior experience with the auditory sequences used in the experimental setting. They had no selfreported history of hearing or psychiatric disorder. The study was conducted in accordance with the declaration of Helsinki, and approved by the Ethics Committee of the University of Leipzig with respect to testing of patients and healthy controls.

\subsection{Auditory stimuli}

The auditory stimuli were created in Audacity 1.2.6 (http:// audacity.sourceforge.net/) and presented binaurally through earphones at a comfortable hearing level (via Presentation Software 14.2, Neurobehavioral Systems, Inc., Berkeley, USA). The audio files are available on the following website (https:// www.dropbox.com/sh/wnn2gzmpwv0u7x3/ AABvk4jCBhP9TT1xpksCh_rba?dl=0). 
Table 1 - Patients' age, and lesions' locations, volume, and date of incident.

\begin{tabular}{|c|c|c|c|c|c|c|c|c|}
\hline & Age & Location & Additional locations & Incident & Volume in cc & $\mathrm{X}$ & $\mathrm{Y}$ & $\mathrm{Z}$ \\
\hline \multirow[t]{11}{*}{ Cerebellar patients } & 64 & posterior cerebellar lobule & none & Nov-06 & 7.1 & 89 & 37 & 26 \\
\hline & 30 & posterior cerebellar lobule & $\begin{array}{l}\text { superior frontal gyrus, corpus } \\
\text { callosum }\end{array}$ & May-98 & 48.0 & 77 & 38 & 35 \\
\hline & 59 & posterior cerebellar lobule, tonsil & none & Jun-07 & 14.5 & 57 & 40 & 24 \\
\hline & 49 & posterior cerebellar lobule, tonsil, vermis & none & Jan-03 & 7.9 & 62 & 41 & 24 \\
\hline & 53 & anterior and medial cerebellar lobule, tonsil & none & unknown & 2.4 & 88 & 52 & 15 \\
\hline & 39 & anterior and posterior cerebellar lobule & none & unknown & 9.0 & 111 & 69 & 37 \\
\hline & 62 & posterior cerebellar lobule & thalamus & May-08 & 3.1 & 57 & 58 & 55 \\
\hline & 62 & posterior cerebellar lobule & telencephalic white matter & unknown & 3.6 & 110 & 38 & 30 \\
\hline & 63 & posterior cerebellar lobule & telencephalic white matter & Jan-07 & 5.7 & 104 & 61 & 46 \\
\hline & 37 & vermis, deep cerebellar nuclei, peduncle & none & unknown & 8.8 & 84 & 49 & 37 \\
\hline & 42 & posterior cerebellar lobule & none & Aug-06 & 0.3 & 121 & 48 & 27 \\
\hline \multirow{11}{*}{$\begin{array}{l}\text { Basal ganglia } \\
\text { patients }\end{array}$} & 41 & putamen, pallidum, caudate body, IC & corona radiata & Dec-98 & 23.4 & 56 & 113 & 76 \\
\hline & 59 & putamen, pallidum, caudate head and body, IC & corona radiata & Apr-05 & 18.3 & 53 & 119 & 76 \\
\hline & 52 & putamen & none & Mar-97 & 2.8 & 104 & 99 & 74 \\
\hline & 55 & putamen, pallidum, caudate body & none & Oct-97 & 3.0 & 102 & 101 & 77 \\
\hline & 61 & $\begin{array}{l}\text { claustrum, putamen, pallidum, caudate body, } \\
\text { EC, IC }\end{array}$ & corona radiata, thalamus & Feb-98 & 8.8 & 51 & 98 & 77 \\
\hline & 37 & putamen, caudate body, IC & none & Apr-03 & 0.8 & 55 & 97 & 88 \\
\hline & 50 & putamen, caudate body, IC & corona radiata, corpus callosum & May-99 & 6.7 & 54 & 110 & 81 \\
\hline & 60 & putamen & none & Jan-89 & 0.5 & 47 & 102 & 64 \\
\hline & 51 & claustrum, putamen, EC & insula & Oct-09 & 14.8 & 106 & 102 & 78 \\
\hline & 64 & putamen, pallidum, caudate body, IC & none & May-07 & 6.1 & 98 & 116 & 80 \\
\hline & 49 & putamen, caudate body & thalamus & Jul-08 & 6.3 & 93 & 103 & 82 \\
\hline
\end{tabular}

Abbreviations: IC = internal capsule, EC = external capsule. 


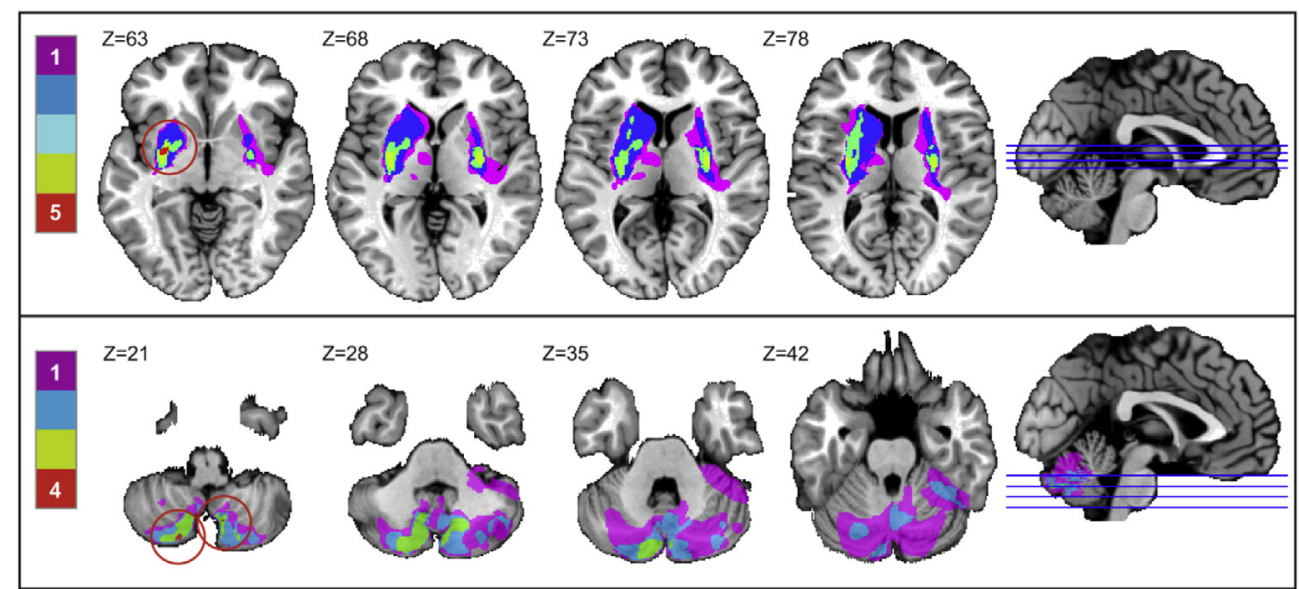

Fig. 1 - Imagery of the lesions. MRIcron (http://www.mccauslandcenter.sc.edu/mricro/mricron/) was used to generate a lesion overlap in the patient groups. Purple shades are associated with minimal lesion overlap $(N=1)$, red shades with maximal lesion overlap (maximum indicated by red circles in the imagery). Upper panel. Basal ganglia lesions (11 patients). Bottom panel. Cerebellar lesions (11 patients).

The stimulus sequences were generated based on two different rhythms continuously looped for $33 \mathrm{sec}$ (Fig. 2). The structure of the rhythms was defined by 12 intervals of $200 \mathrm{msec}$, comprised of sound events and silent intervals (the sounds consisted of $1000 \mathrm{~Hz}$ pure tones, with $10 \mathrm{msec}$ rise and fall times). These rhythms were selected based on previous evidence that they induce a beat at a specific frequency across individuals (Nozaradan et al. 2012b, 2016a). More specifically, these rhythms are known to induce perception of a beat based on a preference for grouping by four elements, at positions indicated in Fig. 2 (i.e. either silent intervals or tone events) with three such beats per rhythm cycle (Nozaradan et al., 2016a). Based on these principles, the first rhythmic sequence is defined as unsyncopated, as a tone event coincides with every beat in most of the possible beat positions, whereas the second rhythm is considered syncopated, as it includes more instances where a tone occurs between beats and is followed by a silent interval on the next beat. The perception of a beat in a syncopated rhythm is correspondingly assumed to rely relatively more on endogenous processes while for the unsyncopated rhythm it presumably reflects relatively more exogenous processes (Fig. 2).

To test whether the expected responses at beat frequency were affected by tempo, participants also listened to the unsyncopated rhythm at three different tempi, including the base tempo as detailed above, as well as at two faster tempi. At the base tempo, the unitary elements in the rhythm (sound events or silent intervals) lasted $200 \mathrm{msec}(5 \mathrm{~Hz})$; at tempo "x2", $100 \mathrm{msec}(10 \mathrm{~Hz})$; and at tempo " $\mathrm{x} 4$ ", $50 \mathrm{msec}(20 \mathrm{~Hz})$. Thus, the duration of one cycle of the rhythm at each of these tempi corresponded to $2400 \mathrm{msec}$ for the base tempo, $1200 \mathrm{msec}$ for tempo $\mathrm{x} 2$, and $600 \mathrm{msec}$ for tempo $\mathrm{x} 4$. As the perceived beat is expected to coincide with the grouping by four unitary elements in this rhythm (Nozaradan et al., 2012b; 2016a) one beat cycle at each of these tempi corresponded to $800 \mathrm{msec}(1.25 \mathrm{~Hz})$ for the base tempo and $400 \mathrm{msec}(2.5 \mathrm{~Hz})$ for tempo x2. However, for tempo $\mathrm{x} 4$, previous work showed that beat perception switches to a larger grouping (12 elements, i.e. $1.6 \mathrm{~Hz}$ beat frequency; Nozaradan et al., 2012b). This switching phenomenon from one temporal grouping of elements to a larger grouping potentially allows the participants to keep the perception of a beat beneath $5 \mathrm{~Hz}$, that is, within the range of tempi observed in music to perceive and synchronize to a beat (van Noorden \& Moelants, 1999; Repp, 2005).

The frequencies at which the neural activity in response to each sequence were expected in the EEG were determined by extracting the temporal envelope of each sequence using a Hilbert function as implemented in Matlab (The MathWorks, USA), yielding a time-varying estimate of the instantaneous amplitude of the sequence envelope (Cirelli, Spinelli, Nozaradan, \& Trainor, 2016; Nozaradan et al., 2016a; Nozaradan, Peretz, \& Keller, 2016b; Nozaradan, Schönwiesner, Caron-Desrochers, \& Lehmann, 2016c). The obtained waveforms were then transformed in the frequency domain using a discrete Fourier transform, yielding a frequency spectrum of envelope magnitude. As shown in Fig. 2, these envelope spectra consisted of a set of 12 distinct harmonic frequencies ranging from the frequency that corresponds to the period of one rhythm cycle to the frequency that corresponds to the period of one element.

\subsection{Experimental conditions and EEG recording}

The four sequences (unsyncopated sequence at base tempo, at tempo $\mathrm{x} 2$, and at tempo $\mathrm{x} 4$, syncopated sequence) were presented in separate blocks. In each block, the 33-sec sequence was repeated 10 times. The onset of each trial was self-paced and was preceded by a $3 \mathrm{~s}$-silent foreperiod. The order of the blocks was always as listed above, and the same for each participant.

Participants were asked to carefully listen to the sequences in order to detect brief changes in the duration of the events (duration of unitary elements reduced or increased by $20 \%$ ), inserted at a random position in two of the trials within each block. Participants were asked to report change detection at 

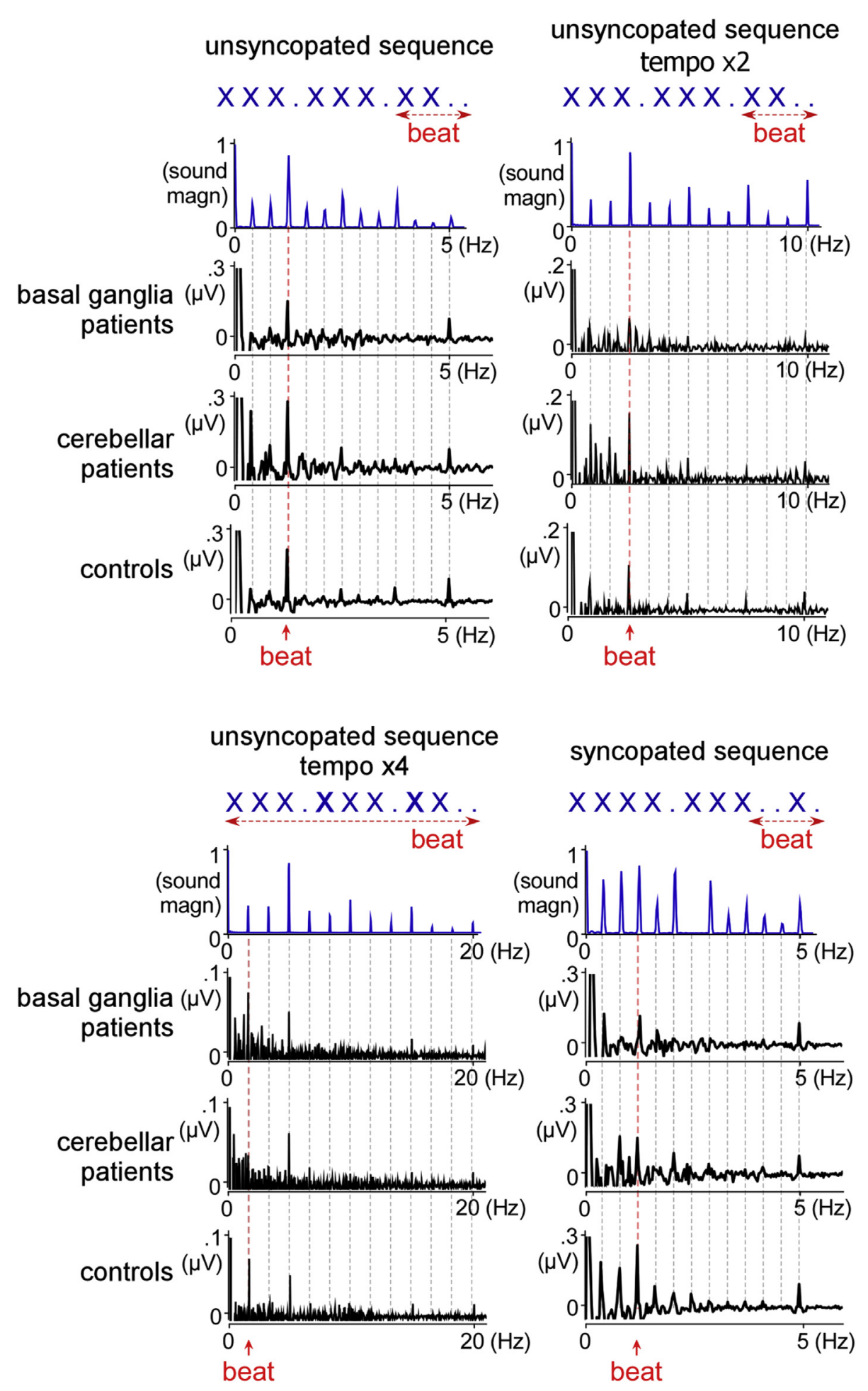

Fig. 2 - Sequences' modulation spectrum (blue) and corresponding EEG spectrum (black). For the three groups of participants, frequency-tagged responses were identified at frequencies corresponding to the frequency components of the rhythmic contour of the sequences. Crosses $=$ sound event; dots = silent interval; in red = theoretical beat frequency (based on previous behavioral data).

the end of each trial. This basic task was not specifically designed to capture behavioral measures of the potential deficits resulting from the lesions. Rather, it ensured that participants remained attentive to the presented sequences and that there was no general hearing impairment in the patients as compared to healthy controls. Task-related trials were excluded from further EEG analyses.

Participants were comfortably seated in a chair with their head resting on a neck support. They were instructed to relax, avoid any head or body movement, and keep their eyes fixated 
on a cross continuously displayed in the center of a screen set in front of them. The electroencephalogram (EEG) was recorded using $59 \mathrm{Ag}-\mathrm{AgCl}$ electrodes placed on the scalp according to the International 10/10 system. Vertical and horizontal eye movements were monitored using four additional electrodes placed on the outer canthus of each eye, and on the inferior and superior areas of the left orbit. Electrode impedances were kept below $5 \mathrm{k} \Omega$. The signals were amplified, lowpass filtered at $512 \mathrm{~Hz}$, digitized using a sampling rate of $1024 \mathrm{~Hz}$, and referenced to an average reference (64-channel high-speed amplifier, Biosemi, The Netherlands).

\subsection{EEG analysis}

The continuous EEG recordings were down-sampled by a factor of two. They were filtered using a $.1 \mathrm{~Hz}$ high-pass Butterworth zero-phase filter to remove very slow drifts from the signals. Up to $5 \%$ of artefact-prone channels were linearly interpolated using pooled neighboring channels. Epochs lasting $32 \mathrm{sec}$ were obtained by segmenting the recordings from +1 to $+33 \mathrm{sec}$ relative to the onset of the trials. The EEG recorded during the first second of each epoch was removed to discard transient auditory evoked potentials related to the onset of the trials (Nozaradan, Peretz, Missal, \& Mouraux, 2011; Nozaradan, Peretz, \& Mouraux, 2012a; Saupe, Schröger, Andersen, \& Müller, 2009).

For each participant and sequence, EEG epochs were averaged across trials ( 8 trials per sequence). This timedomain averaging procedure was used to enhance the signal-to-noise ratio of EEG activity time-locked to the sequences. Averaged waveforms were then transformed to the frequency domain using a discrete Fourier transform, which yielded a frequency spectrum of signal amplitudes $(\mu \mathrm{V})$ ranging from 0 to $256 \mathrm{~Hz}$ with a frequency resolution of $.031 \mathrm{~Hz}$. These EEG processing steps were carried out using Letswave5 (http://nocions.webnode.com/) and Matlab (The MathWorks, USA).

Within the obtained frequency spectra, the signal amplitude can be expected to correspond to the sum of (i) EEG responses elicited by the stimulus, and (ii) unrelated residual background noise. To obtain valid estimates of the responses, the contribution of this noise was minimized by subtracting, at each bin of the frequency spectra, the average amplitude measured at neighboring bins (from bin 3 to 5 relative to each frequency bin) (Chemin, Mouraux, \& Nozaradan, 2014; Mouraux et al., 2011; Nozaradan, Zerouali, Peretz, \& Mouraux, 2015; Wang et al., 2012). To allow the amplitudes of the responses to be compared across participants while avoiding electrode selection bias, the obtained spectra were averaged across all scalp electrodes for each sequence and participant (Fig. 2) (Nozaradan et al., 2011; 2012b; 2016a).

In these noise-subtracted spectra, the magnitude of the twelve peaks of response expected to be elicited by each sequence based on the frequency analysis of the sequences was then estimated by taking the maximum amplitude measured in a range of 3 noise-subtracted frequency bins centered over each of these twelve frequencies. This range allowed accounting for possible spectral leakage due to the fact that the discrete Fourier transform did not estimate signal amplitude at the exact frequency of the expected responses
(Nozaradan et al., 2011; 2012a,b). Finally, the amplitude of the twelve frequency-tagged responses elicited by each sequence was expressed as z-score for each participant, thus allowing the standardized amplitudes to be compared across individuals and sequences (Cirelli et al., 2016; Nozaradan et al., 2012b; 2016a).

\subsection{Statistical analyses}

Statistical analyses were performed using SPSS Statistics 21.0 (IBM, Armonk, NY, USA). The significance level was set at $p<.05$. When relevant, the Greenhouse-Geisser correction was used to correct for violations of sphericity in the performed ANOVA.

We first examined whether each sequence elicited overall significant frequency-tagged responses (Nozaradan et al., 2016a; 2016b). A one-sample t-test was used to determine whether the amplitudes (in $\mu \mathrm{V}$ ) averaged over the 12 expected frequency-tagged responses were significantly different from zero, for each participant and sequence. In the absence of a significant response, these noise-subtracted signal amplitudes may be expected to tend towards zero (Mouraux et al., 2011). We then assessed whether these values were significantly different across the sequences and the three groups of participants, using a repeated-measures ANOVA with 'Sequence' as within-group factor (four levels, for the four different sequences), and a between-group comparison. This allowed testing whether the patients exhibited an overall decrease in the global amplitude of the responses to the auditory inputs when compared to the controls, as an index for the general capacity of the auditory system to respond to the sequences, regardless of the relative amplitude of each frequency component elicited by the rhythmic sequences.

We then tested the specific hypothesis of an altered relative enhancement of the neural response at beat frequency in patients compared to controls. To test this hypothesis, an index of relative amplitude at beat frequency was obtained for each participant by calculating the difference between the amplitude elicited by each sequence at beat frequency and at non-beat frequencies (Nozaradan et al., 2016c). These values were then corrected by subtracting the corresponding standardized values obtained from the sound envelope signals, thus accounting for differences in relative magnitude at beat frequency across sound sequences. Hence, regardless of whether the beat frequency coincided with a prominent frequency component in the sound sequences, a value higher than zero would indicate a relative enhancement of the response at beat frequency. The difference across the three groups in this index averaged across sequences was then estimated using a one-way ANOVA.

Critically, we also hypothesized that, when estimated for each sequence separately, the index of relative amplitude at beat frequency would be significantly reduced in the cerebellar patients, specifically in response to the sequence played at tempo $\mathrm{x} 4$ as this tempo would place highest demands on the temporally precise encoding of the events in the sequence, and require to switch the perceptual organization of the temporal groupings to a lower frequency. In contrast, basal ganglia patients were hypothesized to show reduced relative amplitude at beat frequency, especially in response to the 
syncopated sequence, as this sequence relies relatively more on an endogenous generation of the beat. To test these hypotheses, the index of relative amplitude at beat frequency was compared using an ANOVA with 'Sequence' as withingroup factor (four levels, for the four sequences) and a between-group factor 'Group' (basal ganglia us cerebellar us controls). A significant interaction between the two factors would thus indicate a difference of relative amplitude at beat frequency across sequences and groups. If significant, this interaction was resolved using post-hoc partial ANOVAs and ttests.

\section{Results}

\subsection{Detection task}

All participants correctly detected the targets embedded in the four sequences (i.e. the brief changes in event duration inserted at a random position in two of the trials interspersed within the blocks). In basal ganglia patients, detection performance was $6.63 \pm 1.20$ [mean and standard deviation]/8, with a median number of false alarms of 0 (interquartile range: 0-1). For cerebellar patients the detection performance was $6.36 \pm 2.24 / 8$, with a median number of false alarms of 1 (1-3). In the control group, the detection performance was $7.72 \pm .64 / 8$, with a median number of false alarms of 0 (0-1). A one-way ANOVA revealed no significant difference in the number of correct detections across the three groups $\left(F_{2,30}=2.48, \eta p^{2}=.14, p=.10\right)$. A similar ANOVA performed for the false alarms also revealed no differences across the three groups $\left(F_{2,30}=1.46, \eta p^{2}=.08, p=.24\right)$. This overall absence of significant difference across groups in this basic detection task might thus suggest that all participants followed the task instructions and paid attention to the sequences.

\subsection{Frequency-tagged responses}

We first evaluated whether the patient and control groups showed significant responses to the sequences (see values in Table 2). The corresponding frequency-tagged responses are shown in Fig. 2. On average, the four sequences elicited significant responses at the expected frequencies in both patient groups and in the control group (with fronto-central topography across groups and sequences, as previously found for auditory responses to similar rhythmic inputs; see e.g. Nozaradan et al., 2012b) (Fig. 3). These findings indicate significant overall tracking of the temporal contour of the

\section{EEG responses at beat frequency}
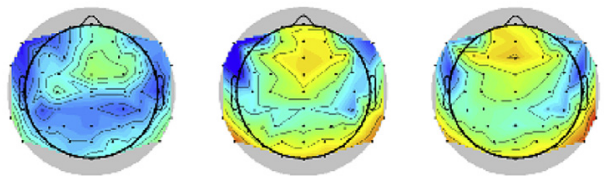

$(\mu \mathrm{V})$

0

\section{EEG responses at non beat frequencies}
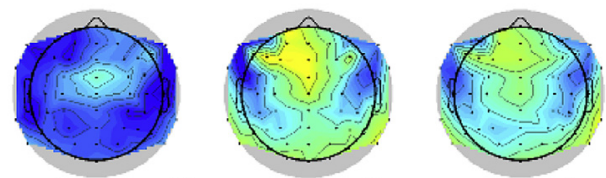

$(\mu \mathrm{V})$

\section{basal ganglia cerebellar controls patients patients}

Fig. 3 - Topographies of the neural activity measured at beat versus non-beat frequencies across the cerebellar patients, basal ganglia patients, and controls (averaged across all sequences).

sequences in the patients and control groups. A repeatedmeasures ANOVA comparing the amplitude of the responses across the four sequences revealed a significant effect of the factor 'Sequence' $\left(\mathrm{F}_{1.82,54.63}=12.99, \eta \mathrm{p}^{2}=.30, p<.0001\right)$ in favor of the two sequences played well within the range of musical tempi (Fig. 2), confirming previous evidence of a preferential frequency range in response to auditory rhythmic input (Nozaradan et al., 2012b; 2016a). However, there was no interaction with the between-group factor $\left(\mathrm{F}_{3.64}=1.39\right.$, $\eta \mathrm{p}^{2}=.08, p=.25$ ). Together with the behavioral measures, this result indicates an overall similar global auditory activity in response to sound sequences across the three groups.

The main goal of the current work though was to estimate the relative amplitude at beat frequency elicited by the sequences in the patients as compared to the controls. The oneway ANOVA used to compare the measures of relative amplitude at beat frequency across groups revealed significant differences $\left(F_{2,30}=9.82, \eta p^{2}=.39, p=.0005\right)$. Specifically, values obtained in the control group were significantly higher than those obtained in the cerebellar or the basal ganglia patient groups $\left(t_{20}=3.37, p=.003\right.$ and $t_{20}=5.30, p<.0001$, respectively), showing an overall reduced relative amplitude at beat frequency in patients (Fig. 4). Moreover, there was no significant difference between the two patient groups $\left(\mathrm{t}_{20}=.685, p=.501\right)$.

Finally, we tested the hypothesis that cerebellar and basal ganglia patients should show differential profiles in response

Table 2 - Mean and standard deviation of the responses to the four sequences

\begin{tabular}{llccc}
\hline & $\begin{array}{c}\text { Unsyncopated } \\
\text { sequence }\end{array}$ & $\begin{array}{c}\text { Unsyncopated sequence } \\
\text { tempo x2 }\end{array}$ & $\begin{array}{c}\text { Unsyncopated sequence } \\
\text { tempo } 4\end{array}$ & Syncopated sequence \\
\hline BG & $.07 \pm .03 \mu \mathrm{V} \mathrm{t}_{10}=6.26^{* * * *}$ & $.04 \pm .02 \mu \mathrm{V} \mathrm{t}_{10} 6.94^{* * * *}$ & $.04 \pm .01 \mu \mathrm{V} \mathrm{t}_{10}=9.03^{* * * *}$ & $.08 \pm .03 \mu \mathrm{V} \mathrm{t} \mathrm{t}_{10}=7.12^{* * * *}$ \\
$\mathrm{CE}$ & $.11 \pm .04 \mu \mathrm{V} \mathrm{t}_{10}=7.41^{* * * *}$ & $.06 \pm .02 \mu \mathrm{V} \mathrm{t} 10=7.82^{* * * *}$ & $.04 \pm .01 \mu \mathrm{V} \mathrm{t}_{10}=7.47^{* * * *}$ & $.10 \pm .05 \mu \mathrm{V} \mathrm{t} \mathrm{t}_{10}=6.68^{* * * *}$ \\
ctrls & $.07 \pm .07 \mu \mathrm{V} \mathrm{t}_{10}=3.43^{* * *}$ & $.05 \pm .03 \mu \mathrm{V} \mathrm{t}_{10}=5.47^{* * *}$ & $.04 \pm .01 \mu \mathrm{V} \mathrm{t}_{10}=8.28^{* * * *}$ & $.12 \pm .13 \mu \mathrm{V} \mathrm{t} \mathrm{t}_{10}=3.21^{* * *}$ \\
\hline
\end{tabular}

Significant responses were overall elicited at frequencies corresponding to the envelope spectrum of the sequences (t-test against zero of the noise-subtracted amplitudes) for the basal ganglia patients (BG), for the cerebellar patients (CE) and for the controls (ctrls). ${ }^{* * *}: p \leq .001$; ${ }^{* * * *}$ : $p \leq .0001$. 


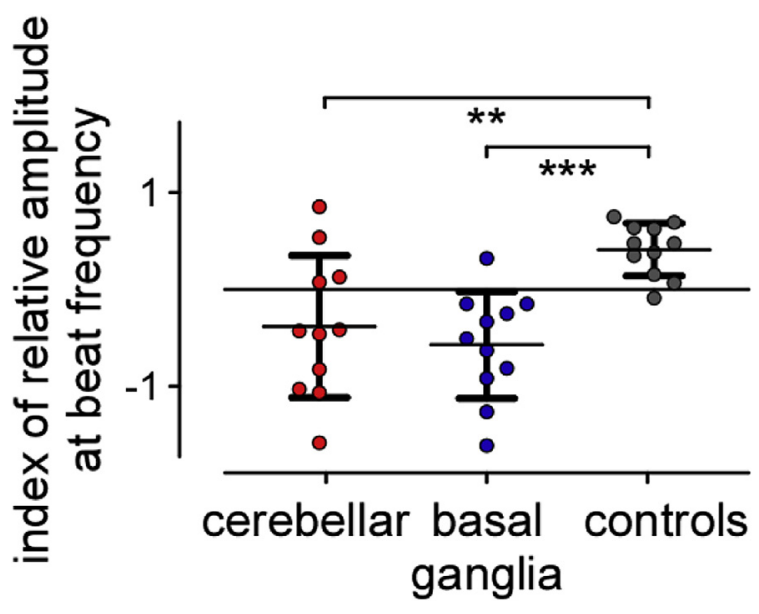

Fig. 4 - Index of relative amplitude at beat frequency averaged across sequences (individual values). There was a significant difference across groups, with both patient groups showing an overall reduced relative amplitude at beat frequency as compared to the controls (asterisks, ttests). ${ }^{* *}: p \leq .01 ;{ }^{* * *}: \leq .001$.

to the beat, which we assumed to be selective to specific sequence types (Fig. 5). To this aim, the index of relative amplitude at beat frequency was compared across groups and sequences using repeated-measures ANOVA. However, as a preceding Levene's test of equality of error variances revealed a significant difference in variability of the distribution across groups specifically for the syncopated sequence $(p=.004)$ but not for all other sequences $(p>.06)$, the ANOVA was performed only on those sequences that passed the equal variance test (unsyncopated sequence at the base tempo, at tempo x2, and at tempo x4). This ANOVA revealed a significant effect of the factor 'Sequence' $\left(F_{1.65,49.64}=89.69, \eta p^{2}=.74\right.$, $p<.0001)$, explained by the difference in tempo across these sequences (Nozaradan et al., 2016a), a significant effect of 'Group' $\left(F_{2,30}=8.05, \eta p^{2}=.34, p=.002\right)$, as well was a significant interaction between the two factors $\left(F_{3.31}=3.59\right.$, $\left.\eta \mathrm{p}^{2}=.19, p=.01\right)$. This analysis revealed that patients displayed different sensitivities to the beat frequency, differing from controls in the processing of specific sequence types.

Post hoc tests were then conducted to compare the groups for each sequence separately using one-way ANOVAs. A significant difference across groups was obtained at the base tempo $\left(F_{2,30}=5.32, \eta p^{2}=.35, p=.01\right)$, and at tempo $\mathrm{x} 4$ $\left(F_{2,30}=4.57, \eta p^{2}=.35, p=.01\right)$, with a similar non-significant trend at tempo $\times 2\left(F_{2,30}=3.03, \eta p^{2}=.21, p=.06\right)$. At base tempo and at tempo $\mathrm{x} 2$, subsequent $t$-tests showed a significantly smaller relative amplitude at beat frequency in basal ganglia patients than controls $\left(\mathrm{t}_{20}=-3.28, p=.004\right.$ and $\mathrm{t}_{20}=2.637, p=.016$, respectively) but not cerebellar patients $\left(t_{20}=1.610, p=.123\right.$ and $t_{20}=.752, p=.461$, respectively). And cerebellar patients did not differ significantly from controls at this base tempo and tempo $\mathrm{x} 2\left(\mathrm{t}_{20}=-1.68, p=.108\right.$ and $t_{20}=1.571, p=.132$, respectively).

In contrast, basal ganglia patients did not differ from controls at tempo $\mathrm{x} 4\left(\mathrm{t}_{20}=.31, p=.76\right)$, whereas cerebellar patients showed a significantly smaller amplitude than controls $\left(\mathrm{t}_{20}=-2.31, p=.03\right)$ and basal ganglia patients $\left(\mathrm{t}_{20}=.752\right.$,
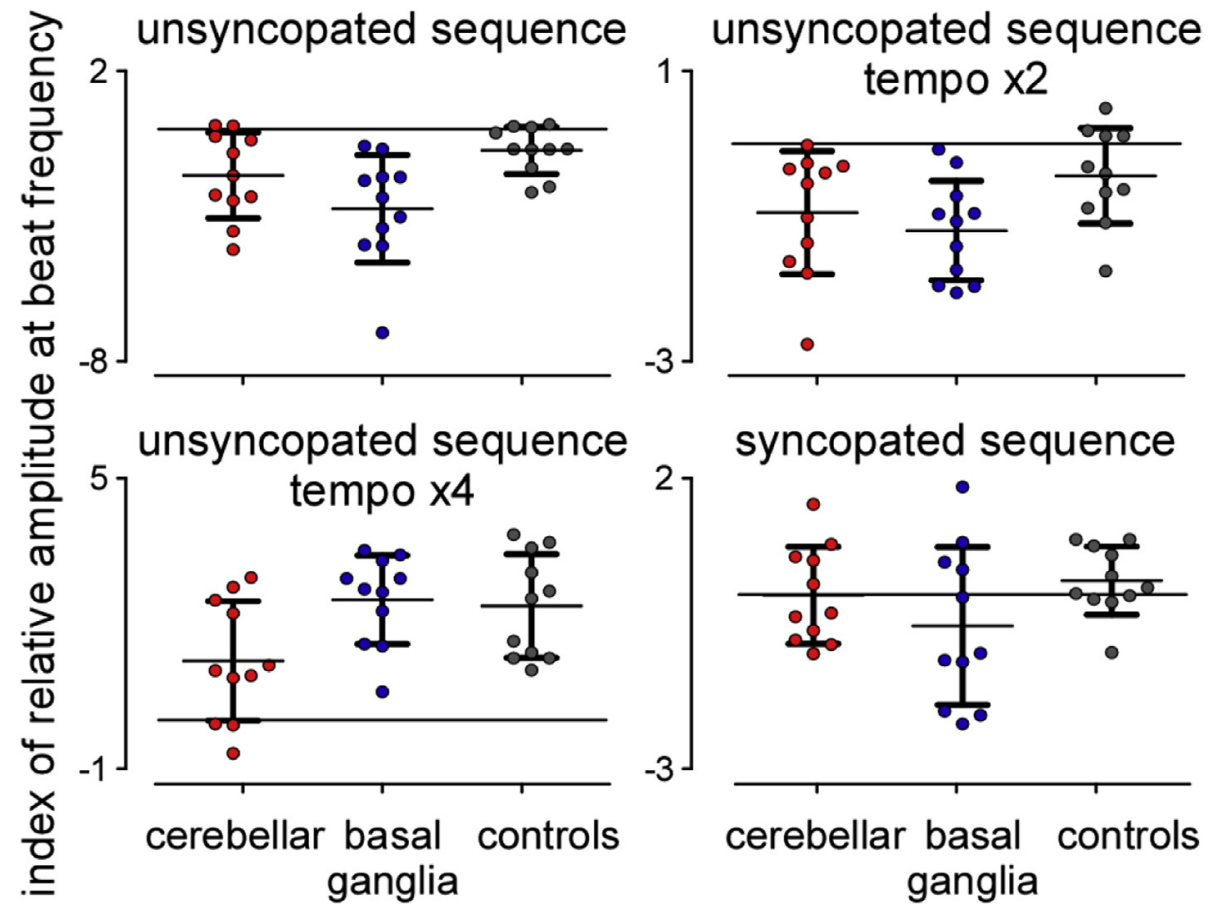

Fig. 5 - Index of relative amplitude at beat frequency for each sequence (individual values). The cerebellar patients exhibited a significantly reduced relative amplitude at beat frequency specifically in response to the sequence played at the fastest tempo (tempo $\mathrm{x} 4$ ) requiring to switch the temporal grouping to a slower frequency. In contrast, the basal ganglia patients exhibited a heterogeneous profile with increased variability in response to the syncopated sequence requiring more internal generation of the beat. 
$p=.460)$. This result confirms the hypothesis of reduced relative amplitude at beat frequency in the cerebellar patients especially at the fastest tempo. This dissociation between the two patient groups also indicates that this effect is not simply an unspecific consequence of stroke lesion but could be indicative of a specific contribution of this brain region to the processing of fast rhythmic sequences.

Although the one-way ANOVA revealed no significant difference across groups for the syncopated sequence $\left(F_{2,30}=1.79, \eta p^{2}=.13, p=.18\right)$, it is not necessarily warranted to conclude that the groups behaved similarly. Indeed, the significant difference in variability across groups provides some indication that at least one of the three groups behaved more heterogeneously when listening to the syncopated sequence. Interestingly this response heterogeneity to the beat was primarily explained by the basal ganglia patients $(-.54 \pm 1.36$ of mean and standard deviation, as opposed to $-.01 \pm .84$ for the cerebellar patients and $.24 \pm .59$ for the control group). This is in line with the hypothesis that basal ganglia patients should display abnormal selective neural entrainment at beat frequency in a rhythm requiring the internal generation of a beat compared to cerebellar patients and healthy controls. Additional non-parametric unpaired Mann-Whitney $U$ tests revealed no significant differences between basal ganglia patients and controls $(p=.131)$, between cerebellar patients and controls $(p=.324)$, and between cerebellar patients and basal ganglia patients $(p=.237)$.

We also tested whether the increased variability in basal ganglia patients for the syncopated sequence could be explained by other variables such as the age of the patients, the number of years since the stroke incident, the lesion volume, and the position of the center of mass of the lesions expressed in stereotactic coordinates (see Supplementary material). However, there was no significant correlation between these measures (all $p$ values $>$.102; Spearman test). Moreover, when divided in two subgroups $(n=5$ for upper values, $n=6$ for lower values), there was no apparent relationship between the lesion topographies and the subgroups (that is, the lesions were mainly overlapping across both groups). Together, these results suggest either a lack of actual covariance between these measures, or an unsufficient number of patients to capture this relationship. Alternatively, it could also suggest that, at least in humans, the processing of more complex rhythms relies on a neural network that goes beyond the ascending auditory pathway and basal ganglia, and is thus not fully accounted for in the lesion topography measures available here.

\section{Discussion}

The current study investigated the functional contribution of the basal ganglia and the cerebellum to the neural representation of rhythmic inputs. To this end, surface EEG activity in response to different rhythmic auditory sequences was compared between healthy controls and patients with lesions in the basal ganglia or the cerebellum. All participants showed significant tracking of the rhythmic input in form of neural responses frequency-locked to the rhythmic contour of the sequences. However, as compared to the controls, patients showed an overall reduced relative amplitude of the neural response at beat frequency. When estimated for each sequence separately, cerebellar patients displayed reduced amplitude at beat frequency in the rhythmic sequence played at the fastest tempo, which requires rapid and temporally precise event coding. In contrast, basal ganglia patients displayed abnormal responses at beat frequency in the more complex sequence that requires relatively more internal generation of the beat as compared to the cerebellar patients and healthy controls.

Whether neural responses to auditory rhythms as recorded with scalp EEG originate from auditory cortical areas or to what extent they constitute a mixture of activities from different cortical and subcortical areas remains unclear. Relatedly, the selective enhancement observed at frequency corresponding to the perceived pulse-like beat in the neural response to rhythms in previous scalp EEG studies could be the product of nonlinear processing arising along the ascending auditory pathway before the auditory cortex in response to sounds (Rajendran, Harper, Willmore, \& Schnupp, 2015). However, a recent study showed that even though part of this neural transform of rhythm could already take place in brainstem auditory loci, processes from the earliest auditory cortical areas of the human brain shape the neural representation of rhythmic inputs in favor of the emergence of a periodic beat (Nozaradan et al., 2016c). This neural transformation of rhythm observed between human primary and secondary auditory cortices in favor of an increased relative prominence of beat frequency is critical especially for more complex rhythms in which the beat frequency is not particularly prominent in the sound envelope spectrum. Yet, these neural responses elicited in auditory cortical areas could be modulated by higher-level associative areas and/or motor areas (Large et al., 2015; Patel \& Iversen, 2014). For instance, recent models proposed that beat perception would involve temporally precise two-way communication between auditory and motor regions in the form of reentrant functional connections (Patel \& Iversen, 2014). According to another model (Large et al., 2015), beat perception would be supported by the selective neural entrainment at beat frequency emerging as the non-linear product of the coupling between the rhythmic input and a network of neural non-linear oscillators embedded in a network of sensory and motor areas. Importantly, by showing a disruption of this process in specific patient groups, the present study shows that this process involves subcortical areas. That is, the processing of auditory rhythms seems to rely on an extended cortico-subcortico-cortical functional network. This network could constitute the actual substrate by which rhythmic perception is coupled to motor entrainment, thus accounting for the modulation of these neural responses to auditory rhythms by body movement (Chemin et al., 2014; Nozaradan et al., 2016b) and also for the inter-individual correlation between these neural responses and motor entrainment to the beat in healthy individuals (Nozaradan et al., 2016a). Hence, although scalp EEG responses to auditory rhythms could originate in major parts from auditory cortical areas (Nozaradan et al., 2015), these responses actually constitute the product of interactive processing stages including the basal ganglia and the 
cerebellum, thus explaining the differences observed across the groups in the current study.

The involvement of subcortical brain areas in rhythm and timing processes has been investigated in a number of neuroimaging studies (Chen, Penhune, \& Zatorre, 2009; Grahn, 2009), particularly pointing to the cerebellum, the basal ganglia, and also the premotor and supplementary motor areas (Chapin et al., 2010; Chen, Penhune, \& Zatorre, 2008; Coull, 2004; Grahn and Brett, 2009; Kung, Chen, Zatorre, \& Penhune, 2013; Penhune, Zattore, \& Evans, 1998; Ramnani \& Passingham, 2001; Schubotz \& von Cramon, 2001; Teki et al.,2011, 2012). However, neuroimaging studies of healthy volunteers are limited in assessing whether these areas are necessary to these processes, and how they specifically contribute to the encoding of external dynamic input.

To overcome this issue, neuropsychological studies have examined temporal processing in patients with damage to certain brain areas. These studies highlighted a similar network of cortical and subcortical areas to that revealed by neuroimaging results of healthy participants (Artieda, Pastor, Lacruz, \& Obeso, 1992; Halsband, Ito, Tanji, \& Freund, 1993; Ivry \& Keele, 1989; Kotz, Stockert, \& Schwartze, 2014; Schwartze et al., 2011; Schwartze, Stockert, \& Kotz, 2015). Hence, converging evidence from multiple methodologies highlights the existence of a functional network of distant brain areas including subcortical areas such as the basal ganglia and the cerebellum in the processing of rhythmic sensory inputs, especially for the encoding of high-level temporal representations.

Yet, the interpretation of patient studies can sometimes be difficult because of task demands that are likely to introduce motivational, decisional, or motor biases in these measures (Grahn, 2009; Rossion, 2014). Moreover, an explicit outcome from the participant may recruit compensatory processes in patients, thus masking the impaired processing. This was the case in a recent study that investigated the ability of patients with lesions in the basal ganglia or in the cerebellum and healthy matched controls to tap in synchrony with adaptive and tempo-changing auditory sequences (van der Steen, Schwartze, Kotz, \& Keller, 2015). Although their movement variability was high, patients generally performed as accurately as controls, thus suggesting the recruitment of compensatory processes related to the explicit task demands imposed on the patients.

Here, we adopted an alternative and more implicit approach to capture the contribution of the target brain areas to the processing of rhythmic input using EEG, i.e., without the need to perform a concomitant explicit behavioral task possibly biasing these measures. Importantly, several points indicate that the differences across patients and healthy controls were unlikely to reflect nonspecific effects. These differences cannot be attributed to a mere impairment in general sensory encoding of the acoustic input in the patients, as the averaged absolute amplitudes of the EEG responses, regardless of the relative amplitude of each frequency component elicited by the rhythmic sequences, were not significantly different across the groups. Also both patients and healthy controls performed similarly in the behavioral auditory detection task. Rather, basal ganglia and cerebellar patients differed in their neural transformation of the rhythms as measured by the relative amplitude at beat frequency. Most importantly, this difference was not observed in all sequences, but was found in the more challenging rhythms involving a relatively increased internal generation of the beat, or a precise encoding of rapid temporal events, respectively. Hence, dissociations across sequences and groups indicate a specific contribution of each of these areas involving specific timing and entrainment sensitivities in the encoding of auditory rhythms. In other words, the results of the current study indicate that the relative contribution of these areas is not intrinsic to the processing of any acoustic input, but might depend on the relative metrical complexity and tempo of the input.

The current results confirm a specific role of the cerebellum in the neural representations of rhythmic sequences, especially those requiring the encoding of precise temporal events in the subsecond range as proposed by the cerebellar timing hypothesis (see e.g. Ivry \& Schlerf, 2008). According to this hypothesis, the cerebellum should specifically engage in automatic event-based temporal processing at short timescales, with sensitivity to changes in sensory inputs occurring in the time-range of 10-30 msec (Buhusi \& Meck, 2005; Coull, Cheng, \& Meck, 2011; Schwartze, Keller, \& Kotz, 2016). The cerebellar neurons would thus transmit a signal in response to the detection of a discrete acoustic event to thalamic and cortical targets and shape the cortical encoding of dynamic auditory input. Interestingly, in the current study, the effect of cerebellar lesions could have been linear across the different tempi. That is, the neural processing of rhythm in cerebellar patients could be gradually disrupted when increasing the tempo. Alternatively, this effect could have been specific to the tempo $\mathrm{x} 4$, as opposed to the base tempo and tempo $\mathrm{x} 2$ sequences. Indeed, previous work has shown that at this fast tempo beat perception switches to the larger grouping of the intervals in the sequence (grouping of 12 elements, i.e. $1.6 \mathrm{~Hz}$ beat frequency; Nozaradan et al., 2012b). In other words, the beat perceived by the participants at this fast tempo corresponds to a slower frequency, even if this slower frequency is not prominent in the sound envelope spectrum of the sequence. This perceptual adjustment potentially allows the participants to keep the perceived beat beneath $5 \mathrm{~Hz}$, that is, within a tempo range allowing body movement synchronization to the beat (van Noorden \& Moelants, 1999; Repp, 2005). Here, the cerebellar patients showed significantly reduced relative amplitude as compared to the controls and the basal ganglia patients, especially in the tempo $\mathrm{x} 4$ sequence, as opposed to the base tempo and tempo x2 sequences. Hence, these results suggest that the effect of cerebellar lesions is not linear across tempi, but appears when the tempo reaches the upper limit of the musical tempo range, thus requiring a perceptual adjustment to process the beat at this fast tempo.

In contrast to the cerebellar patients, the basal ganglia patients displayed altered encoding in response to the rhythms presented well within the musical tempo range for beat perception and production. This was reflected in the unsyncopated rhythm presented at base tempo and at tempo $\mathrm{x} 2$ in which the basal ganglia patients showed a reduced relative amplitude at beat frequency compared to the controls. However, they were not significantly different from cerebellar patients in these conditions, thus suggesting an 
unspecific effect possibly related to suffering from a stroke lesion rather than a specific impairment of this particular brain region. In contrast, in the more complex rhythmic sequence requiring internal generation of the beat, the response at beat frequency was significantly more heterogeneous in the basal ganglia patients as compared to the two other groups. This observation fits well with previous evidence of heterogeneous results gathered in patients with lesions in the basal ganglia specifically in tasks of spontaneous regular tapping (Schwartze et al., 2011). In both cases, the absence of external periodic cues to entrain perception or production required to rely on an internally generated pacing signal. The observed heterogeneity within the basal ganglia patient group especially in this more complex rhythm also suggests possible important inter-individual variability in the outcome of rehabilitation such as auditory-cueing movement training for patients with structural changes in these areas (e.g., Parkinson's disease patients; see Benoit et al., 2014). By characterizing the impact of lesions in this area to the neural representation of syncopated rhythms, the current study thus adds to this line of research investigating the role of the basal ganglia as an internal pacemaker, which could also involve functional connections such as with the pre-supplementary motor area recruited in both rhythmic production and perception (Halsband et al., 1993).

Importantly, in the present study we did not gather explicit behavioral indices of beat perception. Therefore, we cannot ascertain that in these specific patient populations the altered relative amplitude at beat frequency has a direct behavioral relevance for beat processing per se. Nevertheless, recent evidence on a correlation between EEG measures at beat frequency in similar rhythms than those used here and behavioral measures of motor entrainment to the beat and temporal prediction abilities indicates that these neural responses might be somehow related, at least in healthy individuals, to the perceptual phenomenon of beat or underlying mechanisms (Nozaradan et al., 2016a). Future research is needed to directly investigate whether this correlation holds in these patient populations and how lesions in specific parts of the proposed cortico-subcortico-cortical functional network might affect these brain-behavior links. For example, these links could be investigated using frequency-tagging responses during actual sensorimotor synchronization to the beat (see, e.g., Nozaradan et al., 2015; 2016b) or by combining a number of behavioral tests to yield convergent metrics for beat perception to correlate with the EEG measurements.

\section{Conclusions}

The present study provides an original quantification of the neural representation of auditory rhythms across distinct groups of individuals. These responses were characterized by a reduced neural tracking at the beat frequency in patients as compared to healthy controls. It is important to stress that these findings do not imply that these EEG responses constitute a direct neural correlate of beat perception. Rather, these observations suggest that response properties from these subcortical areas may shape the neural representation of rhythmic inputs in favor of the emergence of a periodic beat frequency. Most importantly, these results constitute a critical step forward in our understanding of an extended corticosubcortico-cortical network proposed to support rhythmic sensory processing through differential sensitivities to rhythmic entrainment in this network. But this study calls for future research to clarify the mechanisms by which these neural transformations of rhythm arise in the different regions responding to rhythms and to relate these responses to behavior. Moreover, if the encoding of specific rhythmic input is shown to recruit specific parts of an active auditory-motor neural network, this could open new venues into rehabilitation methods to boost the temporal encoding of rhythmic sounds or the entrainment to move (see e.g. Kotz \& Schwartze, 2010; Schön \& Tillmann, 2015) in patients with sensory or motor disorders, respectively (Bella, Benoit, Farrugia, Schwartze, \& Kotz, 2015; Benoit et al., 2014).

\section{Acknowledgements}

S.N. is supported by the Australian Research Council (DE160101064). This work was also supported by a German Science Foundation Grant to S.A.K (DFG KO2268/6-1).

\section{R E F E R E N C E S}

Artieda, J., Pastor, M. A., Lacruz, F., \& Obeso, J. A. (1992). Temporal discrimination is abnormal in Parkinson's disease. Brain, 115, 199-210.

Bella, S. D., Benoit, C. E., Farrugia, N., Schwartze, M., \& Kotz, S. A. (2015). Effects of musically cued gait training in Parkinson's disease: Beyond a motor benefit. Annals of the New York Academy of Sciences, 1337, 77-85. http://dx.doi.org/10.1111/ nyas.12651.

Benoit, C. E., Dalla Bella, S., Farrugia, N., Obrig, H., Mainka, S., \& Kotz, S. A. (2014). Musically cued gait-training improves both perceptual and motor timing in Parkinson's disease. Frontiers in Human Neuroscience, 8, 494. http://dx.doi.org/10.3389/ fnhum.2014.00494.

Brugge, J. F., Nourski, K. V., Oya, H., Reale, R. A., Kawasaki, H., Steinschneider, M., et al. (2009). Coding of repetitive transients by auditory cortex on Heschl's gyrus. Journal of Neurophysiology, 102(4), 2358-2374.

Buhusi, C. V., \& Meck, W. H. (2005). What makes us tick? Functional and neural mechanisms of interval timing. Nature Reviews. Neuroscience, 6(10), 755-765.

Chapin, H. L., Zanto, T., Jantzen, K. J., Kelso, S. J., Steinberg, F., \& Large, E. W. (2010). Neural responses to complex auditory rhythms: The role of attending. Frontiers in Psychology, 1, 224.

Chemin, B., Mouraux, A., \& Nozaradan, S. (2014). Body movement selectively shapes the neural representation of musical rhythm. Psychological Science, 25(12), 2147-2159.

Chen, J. L., Penhune, V. B., \& Zatorre, R. J. (2008). Listening to musical rhythms recruits motor regions of the brain. Cerebral Cortex, 18(12), 2844-2854.

Chen, J. L., Penhune, V. B., \& Zatorre, R. J. (2009). The role of auditory and premotor cortex in sensorimotor transformations. Annals of the New York Academy of Sciences, 1169, 15-34.

Cirelli, L. K., Spinelli, C., Nozaradan, S., \& Trainor, L. J. (2016). Measuring neural entrainment to beat and meter in Infants: Effects of music background. Frontiers Neuroscience, 10, 229. http://dx.doi.org/10.3389/fnins.2016.00229. 
Coull, J. T. (2004). fMRI studies of temporal attention: allocating attention within, or towards, time. Brain Research, Cognitive Brain Research, 21(2), 216-226.

Coull, J. T., Cheng, R. K., \& Meck, W. H. (2011). Neuroanatomical and neurochemical substrates of timing. Neuropsychopharmacology, 36(1), 3-25.

Ding, N., Melloni, L., Zhang, H., Tian, X., \& Poeppel, D. (2016). Cortical tracking of hierarchical linguistic structures in connected speech. Nature Neuroscience, 19(1), 158-164.

Eggermont, J. J. (2001). Between sound and perception: Reviewing the search for a neural code. Hearing Research, 157(1-2), 1-42.

Gourévitch, B., Le Bouquin Jeannès, R., Faucon, G., \& LiégeoisChauvel, C. (2008). Temporal envelope processing in the human auditory cortex: Response and interconnections of auditory cortical areas. Hearing Research, 237(1-2), 1-18.

Grahn, J. A. (2009). The role of the basal ganglia in beat perception: Neuroimaging and neuropsychological investigations. Annals of the New York Academy of Sciences, 1169, 35-45.

Grahn, J. A., \& Brett, M. (2009). Impairment of beat-based rhythm discrimination in Parkinson's disease. Cortex, 45(1), 54-61.

Grube, M., Cooper, F. E., Chinnery, P. F., \& Griffiths, T. D. (2010). Dissociation of duration-based and beat-based auditory timing in cerebellar degeneration. Proceedings of the National Academy of Sciences of the United States of America, 107(25), 11597-11601. http://dx.doi.org/10.1073/pnas.0910473107.

Grube, M., Lee, K. H., Griffiths, T. D., Barker, A. T., \& Woodruff, P. W. (2010). Transcranial magnetic theta-burst stimulation of the human cerebellum distinguishes absolute, duration-based from relative, beat-based perception of subsecond time intervals. Frontiers in Psychology. http:// dx.doi.org/10.3389/fpsyg.2010.00171.

Halsband, U., Ito, N., Tanji, J., \& Freund, H. J. (1993). The role of premotor cortex and the supplementary motor area in the temporal control of movement in man. Brain, 116, 243-266.

Ivry, R. B.1, \& Keele, S. W. (1989). Timing functions of the cerebellum. Journal of Cognitive Neuroscience, 1(2), 136-152.

Ivry, R. B., Keele, S. W., \& Diener, H. C. (1988). Dissociation of the lateral and medial cerebellum in movement timing and movement execution. Experimental Brain Research, 73(1), $167-180$.

Ivry, R. B., \& Schlerf, J. E. (2008). Dedicated and intrinsic models of time perception. Trends in Cognitive Sciences, 12(7), 273-280.

Joris, P. X., Schreiner, C. E., \& Rees, A. (2004). Neural processing of amplitude-modulated sounds. Physiological Reviews, 84(2), 541-577.

Knolle, F., Schröger, E., Baess, P., \& Kotz, S. A. (2012). The cerebellum generates motor-to-auditory predictions: ERP lesion evidence. Journal of Cognitive Neuroscience, 24(3), 698-706.

Knolle, F., Schröger, E., \& Kotz, S. A. (2013). Cerebellar contribution to the prediction of self-initiated sounds. Cortex, 49(9), 2449-2461.

Kotz, S. A., \& Schwartze, M. (2010). Cortical speech processing unplugged: A timely subcortico-cortical framework. Trends in Cognitive Sciences, 14(9), 392-399.

Kotz, S. A., Stockert, A., \& Schwartze, M. (2014). Cerebellum, temporal predictability and the updating of a mental model. Philosophical Transactions of the Royal Society of London. Series B, Biological Sciences, 369(1658), 20130403.

Kung, S. J., Chen, J. L., Zatorre, R. J., \& Penhune, V. B. (2013) Interacting cortical and basal ganglia networks underlying finding and tapping to the musical beat. Journal of Cognitive Neuroscience, 25(3), 401-420.

Large, E. W., Herrera, J. A., \& Velasco, M. J. (2015). Neural networks for beat perception in musical rhythm. Frontiers in Systems Neuroscience, 25(9), 159. http://dx.doi.org/10.3389/

fnsys.2015.00159.
Liégeois-Chauvel, C., Lorenzi, C., Trébuchon, A., Régis, J., \& Chauvel, P. (2004). Temporal envelope processing in the human left and right auditory cortices. Cerebral Cortex, 14(7), 731-740.

Mouraux, A., Iannetti, G. D., Colon, E., Nozaradan, S., Legrain, V., \& Plaghki, L. (2011). Nociceptive steady-state evoked potentials elicited by rapid periodic thermal stimulation of cutaneous nociceptors. The Journal of Neuroscience, 31, 6079-6087.

van Noorden, L., \& Moelants, D. (1999). Resonance in the perception of musical pulse. Journal of New Music Research, 28, 43-66.

Nozaradan, S. (2014). Exploring how musical rhythm entrains brain activity with electroencephalogram frequency-tagging. Philosophical Transactions of the Royal Society of London. Series B, Biological Sciences, 69(1658), 20130393.

Nozaradan, S., Mouraux, A., Jonas, J., Colnat-Coulbois, S., Rossion, B., \& Maillard, L. (2016a). Intracerebral evidence of rhythm transform in the human auditory cortex. Brain Structure \& Function. http://dx.doi.org/10.1007/s00429-0161348-0.

Nozaradan, S., Peretz, I., \& Keller, P. E. (2016b). Individual differences in rhythmic cortical entrainment correlate with predictive behavior in sensorimotor synchronization. Scientific Reports, 6, 20612. http://dx.doi.org/10.1038/srep20612.

Nozaradan, S., Peretz, I., Missal, M., \& Mouraux, A. (2011). Tagging the neuronal entrainment to beat and meter. The Journal of Neuroscience, 31, 10234-10240.

Nozaradan, S., Peretz, I., \& Mouraux, A. (2012a). Steady-state evoked potentials as an index of multisensory temporal binding. Neuroimage, 60, 21-28.

Nozaradan, S., Peretz, I., \& Mouraux, A. (2012b). Selective neuronal entrainment to the beat and meter embedded in a musical rhythm. The Journal of Neuroscience, 32(49), 17572-17581.

Nozaradan, S., Schönwiesner, M., Caron-Desrochers, L., \& Lehmann, A. (2016c). Enhanced brainstem and cortical encoding of sound during synchronized movement. NeuroImage. pii: S1053-8119(16) 30322-30326.

Nozaradan, S., Zerouali, Y., Peretz, I., \& Mouraux, A. (2015). Capturing with EEG the neural entrainment and coupling underlying sensorimotor synchronization to the beat. Cerebral Cortex. http://dx.doi.org/10.1093/cercor/bht261.

Pantev, C., Hoke, M., Lehnertz, K., Lütkenhöner, B., Anogianakis, G., \& Wittkowski, W. (1988). Tonotopic organization of the human auditory cortex revealed by transient auditory evoked magnetic fields.

Electroencephalography and Clinical Neurophysiolog, 69(2), 160-170.

Patel, A. D., \& Iversen, J. R. (2014). The evolutionary neuroscience of musical beat perception: The action simulation for auditory prediction (ASAP) hypothesis. Frontiers in Psychology, 8, 57. http://dx.doi.org/10.3389/fnsys.2014.00057.

Penhune, V. B., Zattore, R. J., \& Evans, A. C. (1998). Cerebellar contributions to motor timing: A PET study of auditory and visual rhythm reproduction. Journal of Cognitive Neuroscience, 10(6), $752-765$.

Picton, T. W., Skinner, C. R., Champagne, S. C., Kellett, A. J., \& Maiste, A. C. (1987). Potentials evoked by the sinusoidal modulation of the amplitude or frequency of a tone. The Journal of the Acoustical Society of America, 82, 165-178.

Rajendran, V. G., Harper, N. S., Willmore, B. D., \& Schnupp, J. W. H. (2015). A biologically plausible model of beat detection in complex rhythmic sounds. In Proceedings of the rhythm perception and production workshop, Amsterdam, July 2015.

Ramnani, N., \& Passingham, R. E. (2001). Changes in the human brain during rhythm learning. Journal of Cognitive Neuroscience, 13(7), 952-966. 
Repp, B. H. (2005). Sensorimotor synchronization: A review of the tapping literature. Psychonomic Bulletin \& Review, 12, 969-992.

Rorden, C., Karnath, H. O., \& Bonilha, L. (2007). Improving lesionsymptom mapping. Journal of Cognitive Neuroscience, 19(7), 1081-1088.

Rossion, B. (2014). Understanding individual face discrimination by means of fast periodic visual stimulation. Experimental Brain Research, 232(6), 1599-1621.

Saupe, K., Schröger, E., Andersen, S. K., \& Müller, M. M. (2009). Neural mechanisms of intermodal sustained selective attention with concurrently presented auditory and visual stimuli. Frontiers in Human Neuroscience, 3, 58.

Schön, D., \& Tillmann, B. (2015). Short- and long-term rhythmic interventions: Perspectives for language rehabilitation. Annals of the New York Academy of Sciences, 1337, 32-39.

Schubotz, R. I., \& von Cramon, D. Y. (2001). Interval and ordinal properties of sequences are associated with distinct premotor areas. Cerebral Cortex, 11(3), 210-222.

Schwartze, M., Keller, P. E., \& Kotz, S. A. (2016). Spontaneous, synchronized, and corrective timing behavior in cerebellar lesion patients. Behavioural Brain Research, 312, 285-293.

Schwartze, M., Keller, P. E., Patel, A. D., \& Kotz, S. A. (2011). The impact of basal ganglia lesions on sensorimotor synchronization, spontaneous motor tempo, and the detection of tempo changes. Behavioural Brain Research, 216(2), 685-691.

Schwartze, M., \& Kotz, S. A. (2013). A dual-pathway neural architecture for specific temporal prediction. Neuroscience and Biobehavioral Reviews, 37, 2587-2596.

Schwartze, M., Stockert, A., \& Kotz, S. A. (2015). Striatal contributions to sensory timing: Voxel-based lesion mapping of electrophysiological markers. Cortex, 71, 332-340.

van der Steen, M. C., Schwartze, M., Kotz, S. A., \& Keller, P. E. (2015). Modeling effects of cerebellar and basal ganglia lesions on adaptation and anticipation during sensorimotor synchronization. Annals of the New York Academy of Sciences, 1337, 101-110.

Teki, S., Grube, M., \& Griffiths, T. D. (2012). A unified model of time perception accounts for duration-based and beat-based timing mechanisms. Frontiers in Integrative Neuroscience, 5, 90.

Teki, S., Grube, M., Kumar, S., \& Griffiths, T. D. (2011). Distinct neural substrates of duration-based and beat-based auditory timing. The Journal of Neuroscience, 31(10), 3805-3812.

Wang, Y., Ding, N., Ahmar, N., Xiang, J., Poeppel, D., \& Simon, J. Z. (2012). Sensitivity to temporal modulation rate and spectral bandwidth in the human auditory system: MEG evidence. Journal of Neurophysiology, 107(8), 2033-2041. http://dx.doi.org/ 10.1152/jn.00310.2011. 